\title{
A Density Functional Theory Based Electron Transport Study of Coherent Tunneling Through Cyclic Molecules Containing Ru and Os as Redox Active Centers
}

\author{
Xin Zhao $^{1}$ and Robert Stadler ${ }^{1, *}$ \\ ${ }^{1}$ Institute for Theoretical Physics, TU Wien - Vienna University of Technology, \\ Wiedner Hauptstrasse 8-10, A-1040 Vienna, Austria
}

(Dated: December 24, 2018)

\begin{abstract}
In our theoretical study in which we combine a nonequilibrium Green's function (NEGF) approach with density functional theory (DFT) we investigate branched compounds containing Ru or Os metal complexes in two branches, which due to their identical or different metal centers are symmetric or asymmetric. In these compounds the metal atoms are connected to pyridyl anchor groups via acetylenic and phenyl spacer groups in a meta-connection. We find there is no destructive quantum interference (DQI) feature in the transmission function near the Fermi level for the investigated molecules regardless of their symmetry, neither in their neutral states nor in their charged states. We map the structural characteristics of the range of molecules onto a simplified tight-binding model in order to identify the main differences between the molecules in this study and previously investigated ferrocene compounds in order to clarify the structural sources for DQI, which we found for the latter but not for the former. We also find that local charging on one of the branches only changes the conductance by about one order of magnitude which we explain in terms of the spatial distributions and charge-induced energy shifts of the relevant molecular orbitals for the branched compounds.
\end{abstract}

\section{A. Introduction}

Under the restrictions of low bias current flowing through small molecules absorbed to metal electrodes in ultrahigh vacuum at very low temperature, the field of single-molecule electronics $\frac{112}{2}$ has become accessible for a nonequilibrium Green's function (NEGF) approach combined with density functional theory (DFT) ${ }^{3}$, 7 , which allows for an atomic interpretation of experimental results in a mechanical break-junction (MCBJ) or scanning tunneling microscope (STM) setup 8-11. Destructive quantum interference (DQI) effects $12 \mid 13$ allow for the design of logical gates 14 and memory cells ${ }^{15}$ in single molecule electronics as well as the implementation of thermoelectric devices 16[17, since DQI is supposed to significantly reduce the conductance in conjugated $\pi$ systems where such effects were even observed at room temperature 18 .

Experimentally, the design and synthesis of branched compounds containing ferrocene moieties in each branch has been proposed 19] for the purpose of creating single molecule junctions, where the combination of quantum interference effects with redox gating for coherent electron tunneling as well as the electrostatic correlation between spatially distinct redox centers for electron hopping ${ }^{20}$ can be explored. A detailed theoretical analysis of branched compounds containing ferrocene centers has been published in our previous work 22 . In order to assess the generality of this analysis we apply the same models and methods to cyclic $\mathrm{Me}_{2}\left(\mathrm{P}\left(\mathrm{CH}_{3}\right)_{2}\right)_{8}\left(\mathrm{C}_{2} \mathrm{H}_{4}\right)_{4}\left(\mathrm{C}_{6} \mathrm{H}_{4}\right)_{4}$ bis(pyridyl-diacetylide) molecules (Fig. 1), where the two metal atoms $\mathrm{Me}$ are $\mathrm{Ru}$ or $\mathrm{Os}$ and we use the notation of $\mathrm{Ru} / \mathrm{Os}, \mathrm{Os} / \mathrm{Os}, \mathrm{Ru} / \mathrm{Ru}$ for complexes containing symmetrical and asymmetrical branches.

There are experimental and theoretical studies on coherent tunneling and electron hopping through singlebranched molecules containing $\mathrm{Ru}$ atoms as redox-active centers 20|21, where Ref. 20 focuses on the comparison between a coherent tunneling and a hopping mechanism in dependence on the molecular length for ruthenium bis(pyridylacetylide) wires, and the work in Ref. ${ }^{21}$ investigated redox-switches with coherent tunneling for the electron transport through the junction and the switching induced by hopping.

For the work on branched ferrocene compounds we used a simple but effective model, i.e. a combined atomic orbital ( $\mathrm{AO})$ /fragment orbital (FO)-tight-binding (TB) model where we keep only the $p_{z}$ AOs for each atom in both anchoring groups and one relevant bridge FO. For the molecules in Ref. ${ }^{22}$ we found that the throughspace coupling between the two anchor groups is the decisive parameter causing DQI effects in the interesting energy region, i.e. in the gap between the highest occupied molecular orbital (HOMO) and the lowest unoccupied molecular orbital (LUMO) and close to the Fermi level $\mathrm{E}_{F}$. For the molecules which are the focus of this study (Fig. 1) asymmetry can be introduced by exchanging one of the $\mathrm{Ru}$ redox centers with Os, and due to the longer organic bridges, the redox centers are more decoupled from the pyridyl anchors for these molecules than for the ferrocene compounds we studied in 22. In this article, we want to address two issues: i) How do the TB models we used for the branched ferrocene molecules in Ref. ${ }^{22}$ interpret coherent electron transport for this new type of molecules? ii) Will the decreased throughspace couplings due to the longer molecular length have an impact on the occurrence of DQI?

The paper is organized as follows: Section $\mathrm{I}$ gives the computational details for all NEGF-DFT calculations. In section II] we present transmission functions from these calculations for all junction geometries covered in this study and discuss their characteristics features, where comparisons of single and double branched molecules are 


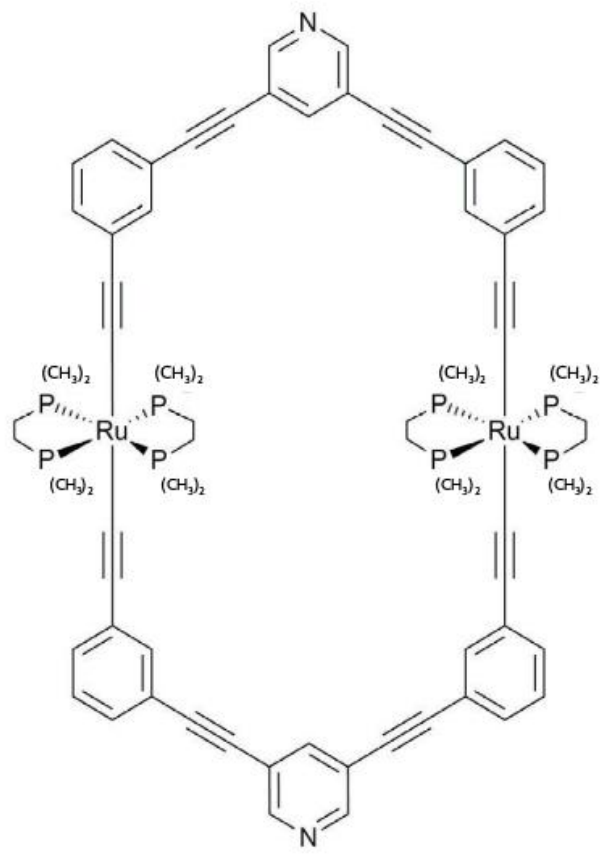

FIG. 1: Chemical structure of a cyclic $\mathrm{Ru}_{2}\left(\mathrm{P}\left(\mathrm{CH}_{3}\right)_{2}\right)_{8}\left(\mathrm{C}_{2} \mathrm{H}_{4}\right)_{4}\left(\mathrm{C}_{6} \mathrm{H}_{4}\right)_{4}$ bis(pyridyl-diacetylide) molecule.

made. In section III we derive topological tight-binding models from the DFT calculations based on the scheme in our previous study where we found DQI for Ferrocene containing compounds and identify the structural sources for the absence of DQI in all Ru, Os containing compounds in the current study. In section IV] we compare the conductance from NEGF-DFT calculations for charged systems with the corresponding neutral ones for assessing the usefulness of these double-branched systems as molecular switches. We conclude with a summary in section $\mathrm{V}$

\section{COMPUTATIONAL DETAILS FOR THE NEGF-DFT CALCULATIONS}

For the computation of transmission probabilities $\mathcal{T}(E)$, we performed DFT calculations with a $\mathrm{PBE}^{23} \mathrm{XC}$ functional within a NEGF framework ${ }^{4 / 7}$ using a linear combination of atomic orbitals (LCAO) 24 as basis set on a double zeta level with polarization functions (DZP) using the GPAW code 25/26, where a grid spacing of 0.2 $\AA$ for the sampling of the potential in the Hamiltonian on a real space grid is used. In our transport calculations, the scattering region is formed by the respective metal organic compounds and three and four layers for the upper and lower fcc gold electrodes, respectively, in a (111) orientation and with $6 \times 10$ gold atoms in the unit cell within the surface plane. The distance between the $\mathrm{Au}$ ad-atom attached to the electrodes surface and the $\mathrm{N}$ atom of the pyridyl anchor groups is $2.12 \AA^{[27}$ and for the $k$ points only the $\Gamma$ point is used in the scattering region for evaluating $\mathcal{T}(E)$ due to the rather large cell sizes in our simulations.

\section{RESULTS AND DISCUSSION OF THE NEGF-DFT CALCULATIONS}

In Fig. 2 we illustrate the molecular junctions derived from the compound in Fig. 1, where we vary the two metal atoms acting as redox-centers to be $\mathrm{Ru} / \mathrm{Os}$, $\mathrm{Os} / \mathrm{Os}$ and $\mathrm{Ru} / \mathrm{Ru}$. In the resulting transmission functions $\mathcal{T}(E)$ for all three combinations and for comparison also compounds with single-branched cases of $\mathrm{Ru}$ and $\mathrm{Os}$, which we show in Fig. 3 , the HOMO peaks are close to the Fermi level for all investigated systems. Hence we expect the conductance to be dominated by the MOs below $E_{F}$.

Our definition of DQI is that the transmission through a system with more than one $\mathrm{MO}$ around $\mathrm{E}_{F}$ is lower than the sum of the individual contributions of these MOs to $\mathcal{T}(E)$ 28/29]. The exact energetic position of the Fermi energy within the HOMO-LUMO gap, which is also affected by the underestimation of this gap in our DFT calculations with a semi-local parametrization of the XC functional, will have a crucial impact on the quantitative value obtained for the conductance but qualitatively DQI will always result in a significant conductance lowering for the structures where it occurs regardless of the details of the Fermi level alignment 29 .

We note that for molecule Ru/Os (green curve, Fig. 3 the peak splitting in the occupied region is distinct due to the intrinsic asymmetry caused by the different metal atoms in the two branches. In order to clarify whether there are DQI effects in the region of the HOMOLUMO gap, we employ a simple TB model with a MO basis, where the eigenenergies of the molecular orbitals and their individual coupling values to the electrodes can be obtained by diagonalizing the subspace of the transport Hamiltonian with the basis functions centered on the molecule ${ }^{22 \mid 30}$. We use Larsson's formula ${ }^{31 \mid 34}$ to calculate an approximation for the transmission function $\mathcal{T}(E)$, where only the frontier orbitals, namely the HOMO and the LUMO are included. As one can see from Fig. 4 the contributions from only the frontier molecular orbitals reproduce the characteristic features (blue curve) of the DFT result (red curve) for molecule $\mathrm{Ru} / \mathrm{Ru}$. From Fig. 4 we also see that when the HOMO and LUMO both are coupled to the electrodes $\left((\mathrm{HOMO}+\mathrm{LUMO})^{2}\right.$, blue curve) we achieve identical result as from the individual 

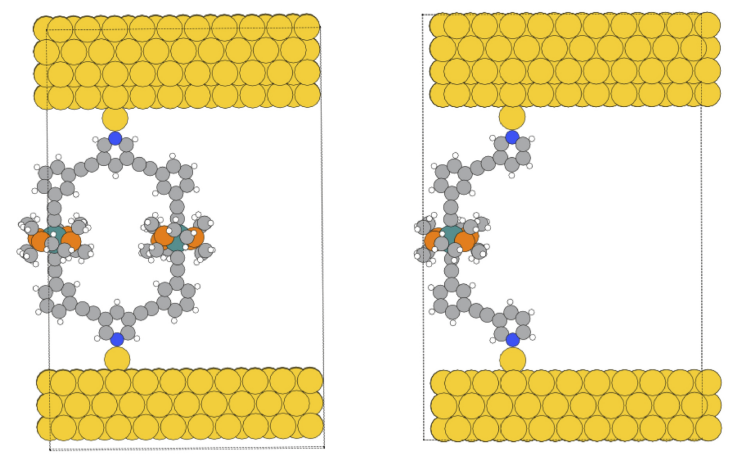

FIG. 2: Junction setups containing the molecule in Fig. 1 where for the double-branched molecule (left panel) the two metal atoms are varied as $\mathrm{Ru} / \mathrm{Ru}, \mathrm{Ru} / \mathrm{Os}$ and Os/Os and for the single branched molecule (right panel) the single metal atom is either $\mathrm{Ru}$ or Os.

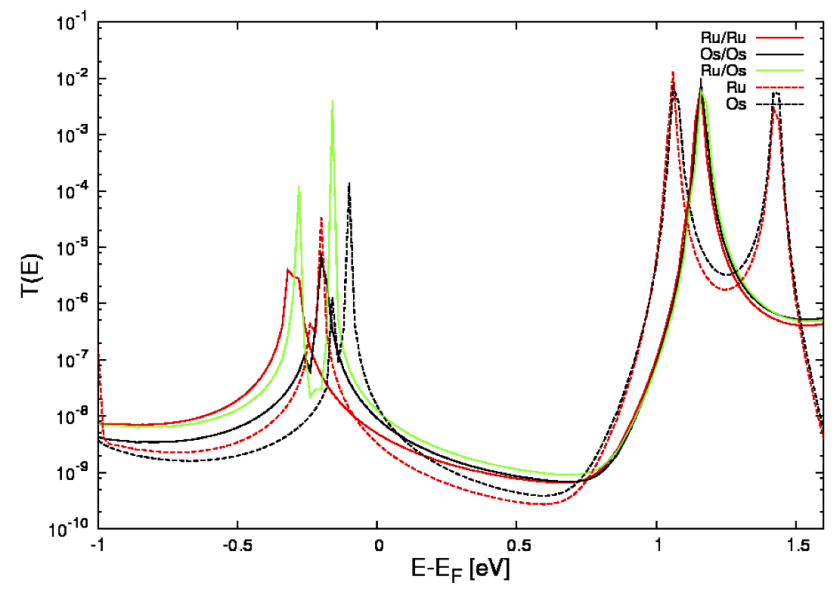

FIG. 3: Transmission functions from NEGF-DFT calculations for the double-branched molecules $\mathrm{Ru} / \mathrm{Ru}$ (solid red line), Os/Os (solid black line), Ru/Os (green solid line) as well as for single-branched $\mathrm{Ru}$ (dashed red line) and Os (dashed black line) in the junction setup shown in Fig. 2 in their neutral states, respectively.

contributions $\left(\mathrm{HOMO}^{2}+\mathrm{LUMO}^{2}\right.$, cyan curve) around $\mathrm{E}_{F}$, which means no DQI of electron transport through these two MOs is occurring for the system $\mathrm{Ru} / \mathrm{Ru}$. For all other systems, namely Ru/Os, Os/Os, single-branched $\mathrm{Ru}$ and $\mathrm{Os}$, we obtain the same results (not shown here).

We also compare the transmission functions for single (red and black dashed lines) and double-branched (red and black solid lines) molecules to illustrate the impact of the number of branches in Fig. 3 . As we can see the transmission functions for the $\mathrm{Ru}$ (dashed red line) and $\mathrm{Ru} / \mathrm{Ru}$ (solid red line) molecules are rather similar, and the zero-bias conductances differ only by a factor

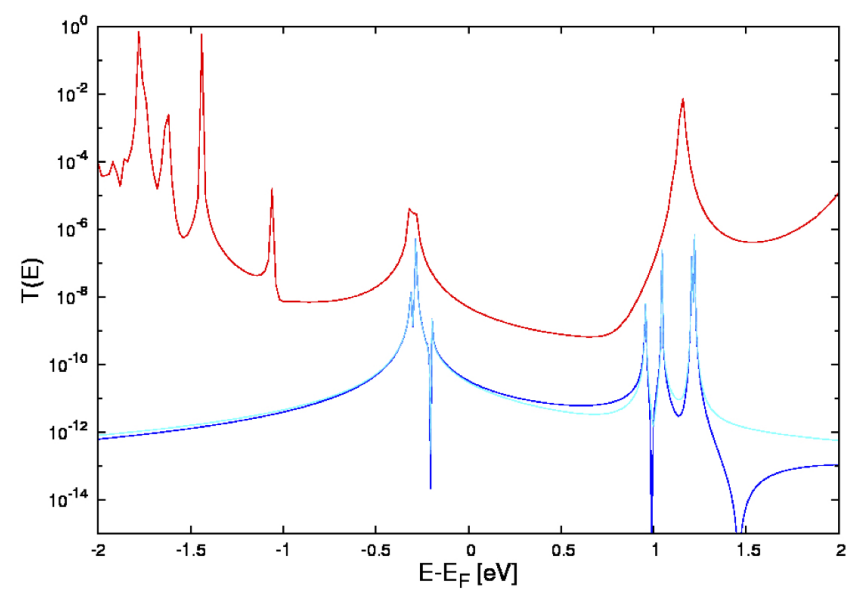

FIG. 4: Transmission functions calculated from NEGF-DFT (red curve) and Larsson's formula for the system $\mathrm{Ru} / \mathrm{Ru}$, where for the latter the blue curve denotes (HOMO + LUMO $)^{2}$ and the cyan curve $\mathrm{HOMO}^{2}+\mathrm{LUMO}^{2}$.

of about 1.5. The amount of peaks in the occupied region for the double-branched molecule is higher than the one in the single-branched molecule as there are more molecular states coupled to the electrodes for the former. Comparing Os (dashed black line) and Os/Os (solid black line), we find the same qualitative differences but the conductance for the double-branched molecule is now only higher by about a factor of 0.6 for the single-branched molecule.

The next question is then what is the reason for the absence of DQI in the HOMO-LUMO gap when these molecules share the design ideas with the ferrocene molecules we studied before 22 . In order to address this question we apply the AO-FO model we previously used for the ferrocene systems ${ }^{22}$ in the next section and take the single-branched $\mathrm{Ru}$ molecule as an example due to its representative transmission function, which is very similar to those of the double-branched $\mathrm{Ru} / \mathrm{Ru}, \mathrm{Os} / \mathrm{Os}$, $\mathrm{Ru} / \mathrm{Os}$ and single-branched Os molecules.

\section{COMPARISON OF SIMPLIFIED $3 \times 3$ HAMILTONIANS FOR SINGLE- BRANCHED FERROCENE AND RUTHENIUM CONTAINING MOLECULES}

Based on the scheme we developed in Ref. ${ }^{22}$ we use the simplified $3 \times 3$ Hamiltonian in order to obtain a mathematical perspective for explaining the role of essential structural features for the occurrence or absence of DQI according to the following procedure, where we take single-branched $\mathrm{Ru}$ as an example. In a first step we define the $p_{z}$ AOs of the anchor groups containing pyridyl and the attached acetylene moieties by diagonalizing the subspace of the transport Hamiltonian corresponding to each carbon or nitrogen atom on these groups and picking 


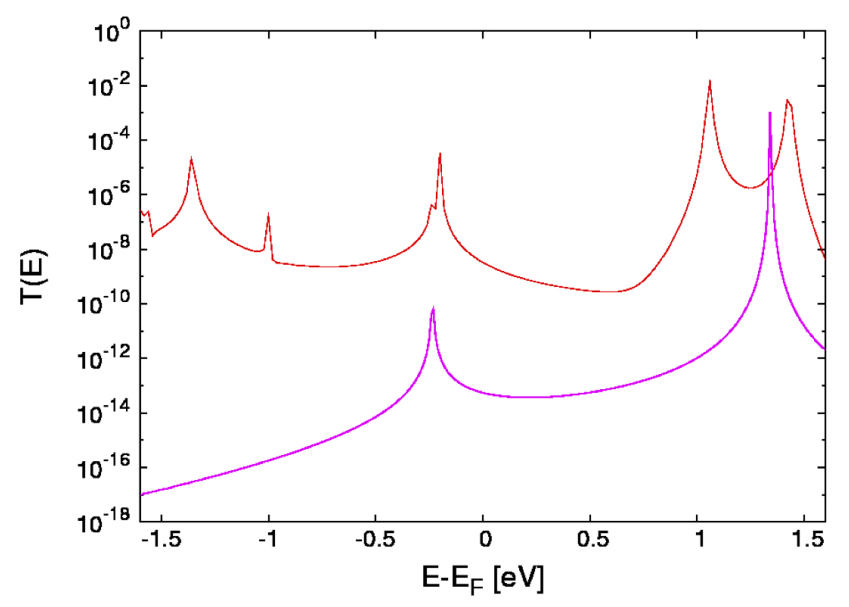

FIG. 5: Transmission functions for a single-branched $\mathrm{Ru}$ molecule, where the red curve is from a NEGF-DFT calculation. The magenta curve is obtained from a NEGF-TB calculation with one FO anchor state on each side and one bridge FO, where the anchor FO is obtained from a subdiagonalization of the transport Hamiltonian containing the $p_{z}$ state of each carbon or nitrogen atom. For the electrodes a chain of single AOs with onsite energies of $0.83 \mathrm{eV}$ and coupling values of $-5.67 \mathrm{eV}$ within the electrodes is used.

the $p_{z}$ states, which can be identified by their onsite energies and symmetry. For the bridge group two relevant bridge FOs (again obtained by a subdiagonalisation) in the occupied region are considered where we define $\mathrm{Ru}$ plus Phosphine ligands and conjugated spacers including the acetylene and benzene groups as part of the bridge. Then we diagonalize the two anchor group subspaces now defined only by $\mathrm{p}_{z}$ orbitals in order to get the relevant anchor FOs on both sides. We then minimize the Hamiltonian to the most simple one, which only contains the three most relevant states, i.e. one FO on each anchor group and one bridge FO. We show $\mathcal{T}(E)$ obtained from NEGF-TB calculations for such a 3 FO model in Fig. 5 , and find that if qualitatively reproduces the characteristic features found in NEGF-DFT calculations.

The most distinct differences between the molecules in Fig. 11 and those studied in Ref. 22 are the molecular length and the type of metal centers. In our previous work ${ }^{22}$ we found that in order to observe a DQI feature close to $\mathrm{E}_{F}$, the through-space coupling $t_{D}$ needs to be neither too small nor too big in size so that the DQI feature will not be pushed outside the relevant energy region around $\mathrm{E}_{F}$.

For a direct comparison of the molecules in our present study with those from our previous study we define the 3 $\times 3$ Hamiltonian obtained from the 3 FO model described above as

$$
H_{m o l}=\left[\begin{array}{ccc}
\varepsilon_{L} & t_{L} & t_{D} \\
t_{L} & \varepsilon_{B} & t_{R} \\
t_{D} & t_{R} & \varepsilon_{R}
\end{array}\right]
$$

TABLE I: Parameters entering the $3 \times 3$ Hamiltonian formed by three FOs ( Fig. 6) for three single-branched systems, where all values are given in $\mathrm{eV}$. The values for Fc are obtained from the calculations presented in Ref. 22 .

\begin{tabular}{cccc}
\hline \hline & $\mathrm{Ru}$ & $\mathrm{Os}$ & $\mathrm{Fc}$ \\
\hline $\mathrm{t}_{L}$ & -0.026 & -0.023 & 0.27 \\
\hline $\mathrm{t}_{R}$ & 0.019 & 0.019 & -0.22 \\
\hline $\mathrm{t}_{D}$ & $5.7 \mathrm{E}-05$ & $-5.1 \mathrm{E}-05$ & -0.023 \\
\hline$\Delta \varepsilon$ & -1.5 & -1.5 & 0.6 \\
\hline \hline
\end{tabular}

for the single-branched $\mathrm{Ru}, \mathrm{Os}$ and ferrocene (Fc) systems in Table I, where $\varepsilon_{L, B, R}$ are the respective onsite energies of the three FOs, $t_{L, R}$ the electronic couplings between the two anchor FOs and the bridge FO and $t_{D}$ the direct coupling between the anchor FOs.

We can see that the coupling values of $t_{L}, t_{R}$ and $t_{D}$ connecting the three FOs for the three systems $\mathrm{Ru}$, Os and $\mathrm{Fc}$ differ in: i) the size of the couplings $\mathrm{t}_{L / R}$ between anchor and bridge, which are one magnitude smaller for $\mathrm{Ru}$ and Os compared with the ones in Fc; ii) the throughspacing coupling $\mathrm{t}_{D}$, which are three magnitudes smaller for the $\mathrm{Ru}$ and Os molecules, and iii) the onsite energy $\varepsilon_{B}$ of the bridge FO we used for the $3 \times 3$ Hamiltonian, which is the highest-lying FO in the occupied region for $\mathrm{Ru}$ and $\mathrm{Os}$ and was the lowest-lying FO in the unoccupied region for Fc. As a result the energy difference between anchor and bridge states $\Delta \varepsilon$ are larger in size for $\mathrm{Ru}$ and Os and the sign differs compared with the ferrocene molecule.

The differences in couplings can be interpreted by visualizing the FOs in Fig. 6. where the symmetry of the FOs on the anchors in Fig. 6(a) for the Ru molecule is equivalent to what is found for the ferrocene molecule (Fig. 6 b)), but $\mathrm{t}_{L / R}$ is decreased markedly, because in between the metal complex and the pyridyl group there is now a benzene unit separating the two for the Ru molecule. The benzene groups are defined as part of the bridge in our subdiagonalization but the resulting bridge FOs close to $\mathrm{E}_{F}$ show no localization on them. In addition, the increased length also strongly reduces the direct coupling $t_{D}$ between the two anchor groups. The bridge state on the $\mathrm{Ru}$ molecule is also localized on the adjacent triple bonds, while for Fc the state is confined to the ferrocene moiety.

Having established above that the parameters $\mathrm{t}_{L / R}, \mathrm{t}_{D}$ and $\Delta \varepsilon$ distinguish the ferrocene molecule Fc with a DQI feature close to the LUMO from the single-branched systems $\mathrm{Ru}$ and $\mathrm{Os}$, we want to further explore the relative importance of these parameters, where we focus on the comparison of $\mathrm{Ru}$ and $\mathrm{Fc}$ regarding the three parameters entering the Hamiltonian. If we mark the three parameters for ferrocene molecule $\mathrm{Fc}$ as $\mathrm{F}_{1}, \mathrm{~F}_{2}, \mathrm{~F}_{3}$ and for $\mathrm{Ru}$ 


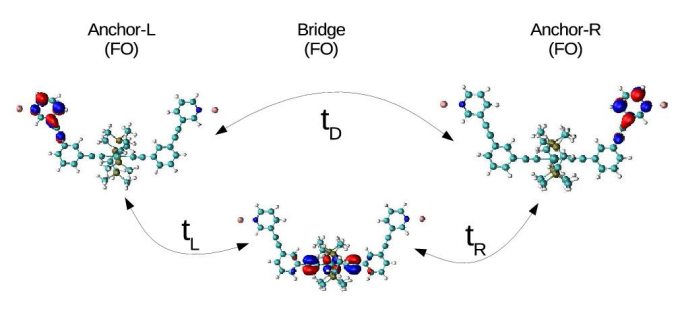

(a)

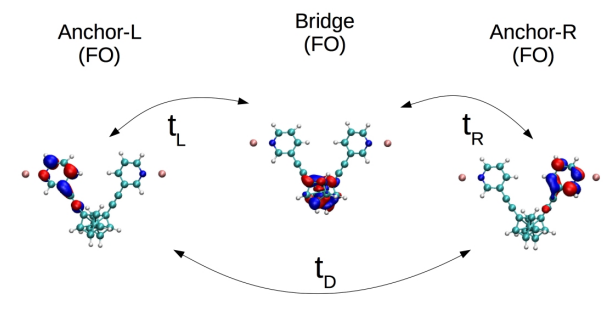

(b)

FIG. 6: Spatial distributions of the three FOs in the simplified model for a) single-branched $\mathrm{Ru}$ with the anchor $\mathrm{FO}$ on each side at $1.3 \mathrm{eV}$ and the bridge $\mathrm{FO}$ at $-0.2 \mathrm{eV}$; b) single-branched $\mathrm{Fc}$ with the anchor $\mathrm{FO}$ on each side at 1.05 $\mathrm{eV}$ and the bridge $\mathrm{FO}$ at $1.66 \mathrm{eV}^{[22}$.

TABLE II: The three parameters defining the $3 \times 3$ Hamiltonian within the 3FO-model which qualitatively reproduce the transmission function for the single-branched $\mathrm{Ru}$ and $\mathrm{Fc}$ systems, respectively.

\begin{tabular}{ccc}
\hline \hline & $\mathrm{Ru}$ & $\mathrm{Fc}$ \\
\hline$\Delta \varepsilon($ parameter 1$)$ & -1.5 & 0.6 \\
\hline $\mathrm{t}_{L / R}($ parameter 2$)$ & 0.025 & 0.25 \\
\hline $\mathrm{t}_{D}($ parameter 3$)$ & $5.0 \mathrm{E}-05$ & -0.023 \\
\hline $\mathrm{t}_{D} / \mathrm{t}_{L / R}$ & $-2.0 \mathrm{E}-03$ & -0.092 \\
\hline
\end{tabular}

as $\mathrm{R}_{1}, \mathrm{R}_{2}, \mathrm{R}_{3}$, where the numbers refer to those given in Table [II] there are six possible combinations of them for forming a $3 \times 3$ Hamiltonian. From these Hamiltonians we calculate the transmission functions with NEGF-TB for each combination in order to identify the decisive parameters enabling DQI.

For the sake of simplicity we approximate the coupling values $\mathrm{t}_{L / R}$ with $0.025 \mathrm{eV}$ and $0.25 \mathrm{eV}$ for $\mathrm{Ru}$ and $\mathrm{Fc}$, respectively, and plot the transmission functions for the six resulting Hamiltonians based on the different combinations of the three parameters in Fig. 7.

As one can see modifying any one of the three parameters in the Hamiltonian of Fc leads to the disappearance of the DQI feature in the interesting region, which indicates that all three parameters have a fundamental influ-

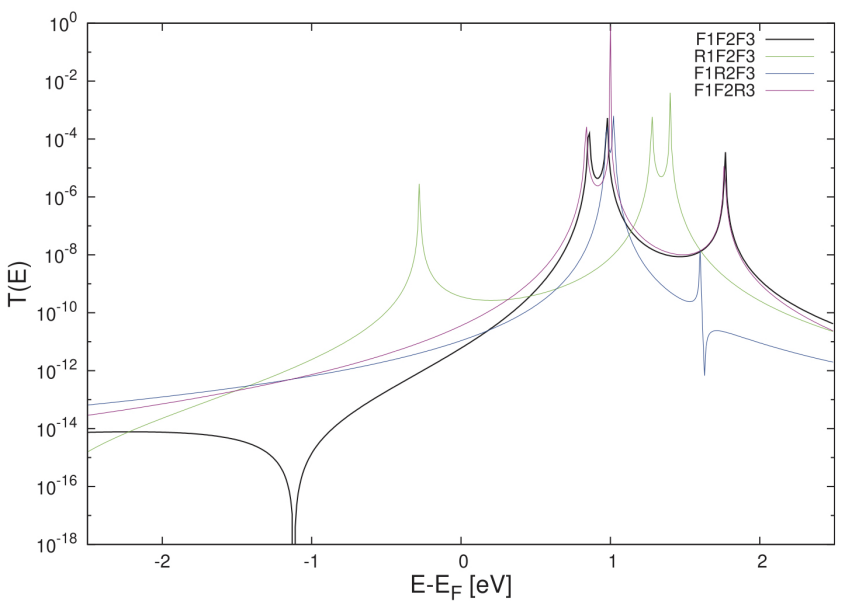

(a)

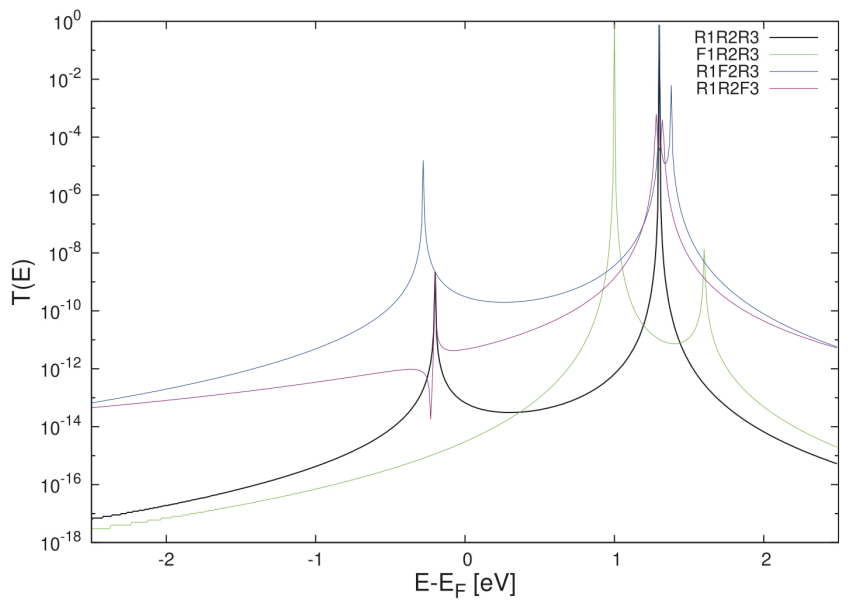

(b)

FIG. 7: Transmission functions obtained from NEGF-TB calculations using the 3FO-model of the single-branched a) Fc molecule (black solid line) and b) Ru molecule (black solid line), where the symbols $\mathrm{F}$ and $\mathrm{R}$ represent $\mathrm{Fc}$ and $\mathrm{Ru}$, respectively, and the indices 1-3 refer to the numbers in

Table II. In panel a) we start from the parametrization of Fc in Table II $\left(\mathrm{F}_{1} \mathrm{~F}_{2} \mathrm{~F}_{3}\right)$ and replace each of the parameters individually by the one corresponding to $\mathrm{Ru}$, and calculate

$T(E)$ for $\mathrm{R}_{1} \mathrm{~F}_{2} \mathrm{~F}_{3}$ (green line), $\mathrm{F}_{1} \mathrm{R}_{2} \mathrm{~F}_{3}$ (blue line) and

$\mathrm{F}_{1} \mathrm{~F}_{2} \mathrm{R}_{3}$ (cyan line). In panel $\mathrm{b}$ ) we permute the parameters in the opposite direction starting from the parametrisation of $\mathrm{Ru}$ in Table $\mathrm{II}\left(\mathrm{R}_{1} \mathrm{R}_{2} \mathrm{R}_{3}\right)$ and obtaning $T(E)$ for $\mathrm{F}_{1} \mathrm{R}_{2} \mathrm{R}_{3}$ (green line), $\mathrm{R}_{1} \mathrm{~F}_{2} \mathrm{R}_{3}$ (blue line) and $\mathrm{R}_{1} \mathrm{R}_{2} \mathrm{~F}_{3}$ (cyan line).

ence on the DQI absence for Ru in the relevant energy region and its their interplay which is decisive for the absence or occurrence of DQI features within the HOMOLUMO gap. The through-space coupling $\mathrm{t}_{D} \mathrm{i}$, however is still special in the sense that taking the value from $\mathrm{Fc}$ in the $\mathrm{Ru}$ Hamiltonian $\left(\mathrm{R}_{1} \mathrm{R}_{2} \mathrm{~F}_{3}\right.$ in Fig. $\left.7 \mathrm{~b}\right)$, induces a DQI minimum slightly below the HOMO peak.

In the following we keep two parameters fixed and vary one in a systematic way to further investigate the role 
each parameter plays. In Fig. 8 we illustrate the relation between each of these three parameters and the energy position of the DQI-induced minimum $\mathrm{E}_{0}$, which we obtain from the eigenenergies and amplitudes at the contact sites of the three MOs resulting from a diagonalization of the parametrized $3 \times 3$ Hamiltonian corresponding to the 3 FO-model in combination with Larsson's formula 229 using the procedure described in detail in Ref. 22].

The such obtained single parameter dependencies can be summarized as linear for $\mathrm{E}_{0}(\Delta \varepsilon)(\mathrm{Fig} 8 \mathrm{~B})$, as quadratic for $\mathrm{E}_{0}\left(\mathrm{t}_{L / R}\right)$ (Fig 8b), and as multiplicative inverse for $\mathrm{E}_{0}\left(\mathrm{t}_{D}\right)$ (Fig 8p). The black curve in Fig. 8a illustrates that for the single-branched $\mathrm{Ru}$ system with $\mathrm{t}_{D}$ and $\mathrm{t}_{L / R}$ fixed to the values in Table III $\mathrm{E}_{0}$ lies always above $\sim 12 \mathrm{eV}$ regardless of the variation of $\Delta \varepsilon$. For the $\mathrm{Fc}$ system (red curve in Fig. $8 \mathrm{E}_{0}$ is around $-1.1 \mathrm{eV}$, i.e. close to $\mathrm{E}_{F}$, for $\Delta \varepsilon$ chosen as in the real system but is pushed below $-3 \mathrm{eV}$ when the value is replaced by the one corresponding to the $\mathrm{Ru}$ molecule.

While $\mathrm{E}_{0}$ shows a significant dependence on $\mathrm{t}_{L / R}$ in Fig $8 \mathrm{~b}$ for both systems with a maximum at $\mathrm{t}_{L / R}=0$, they differ strongly in the sense that this maximum which is defined by $\varepsilon_{B}$ corresponds to the LUMO peak for Fc and the HOMO peak for $\mathrm{Ru}$, meaning that a variation of $\mathrm{t}_{L / R}$ only allows to introduce a DQI minimum into the HOMO-LUMO gap for the former but not for the latter. A variation of the parameter $t_{D}$ on the other side allows for $\mathrm{E}_{0}$ to cross the HOMO-LUMO gap for both systems as ca be seen from Fig 8p albeit for values about an order of magnitude smaller for $\mathrm{Ru}$ when compared with $\mathrm{Fc}$ and for different signs.

We put our findings on the $\mathrm{t}_{D}$ dependence of $\mathrm{E}_{0}$ to a test by performing NEGF-TB calculations for a range of values of $\mathrm{t}_{D}$ where $\Delta \varepsilon$ and $\mathrm{t}_{L / R}$ have been kept fixed to the values of the Ru Hamiltonian in Table II In Fig. 9a we show that the real value for $\mathrm{Ru}$ in Table II $(0.00005$ $\mathrm{eV}$ ) is too small to cause DQI anywhere near the gap, a value largeer by about one order of magnitude (0.0004 $\mathrm{eV}$ ) makes a DQI feature appear above the LUMO and if the value is too large $(0.09 \mathrm{eV})$, the feature merges with the HOMO peak. An intermediate value (0.0002 $\mathrm{eV}$ ) places the DQI induced minimum optimally within the gap and close to the Fermi level, where its influence on the conductance will be most pronounced. In Fig. $9 \mathrm{~b}$ we confirm the findings of Fig 8k, namely that the sign of $\mathrm{t}_{D}$ matters significantly for the location of $\mathrm{E}_{0}$.

For a physical interpretation of our results we note that the ratio $\mathrm{t}_{D} / \mathrm{t}_{L / R}$ we obtain from the optimal $\mathrm{t}_{D}$ value in Fig. $9 \mathrm{a}(0.002 \mathrm{eV})$ is 0.08 meaning that its magnitude is very close to that for the Fc system and about two orders of magnitude larger than the real value of $t_{D}$ for the $\mathrm{Ru}$ molecule in Table II It is intuitively plausible that this ratio plays such a significant role for the occurrence of DQI, where since it is a wave phenomenon destructive interference needs two different path ways which are highly asymmetrical with respect to each other but not more than one order of magnitude apart in their respective couplings. In our model these two path ways are

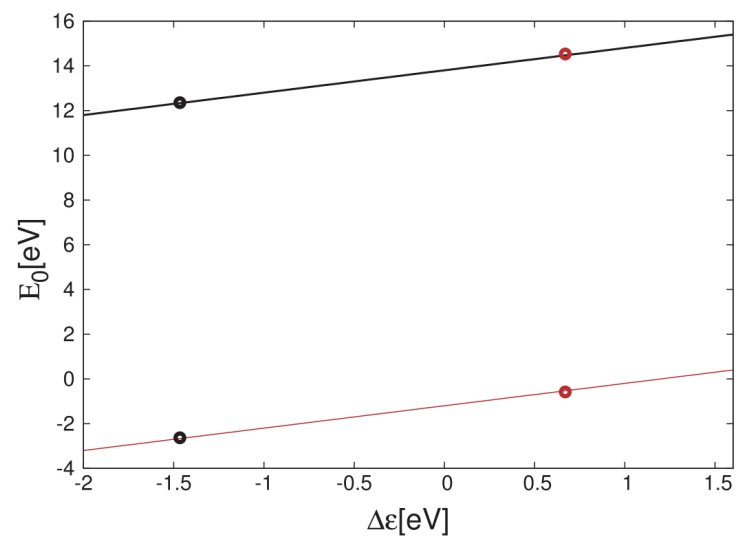

(a)
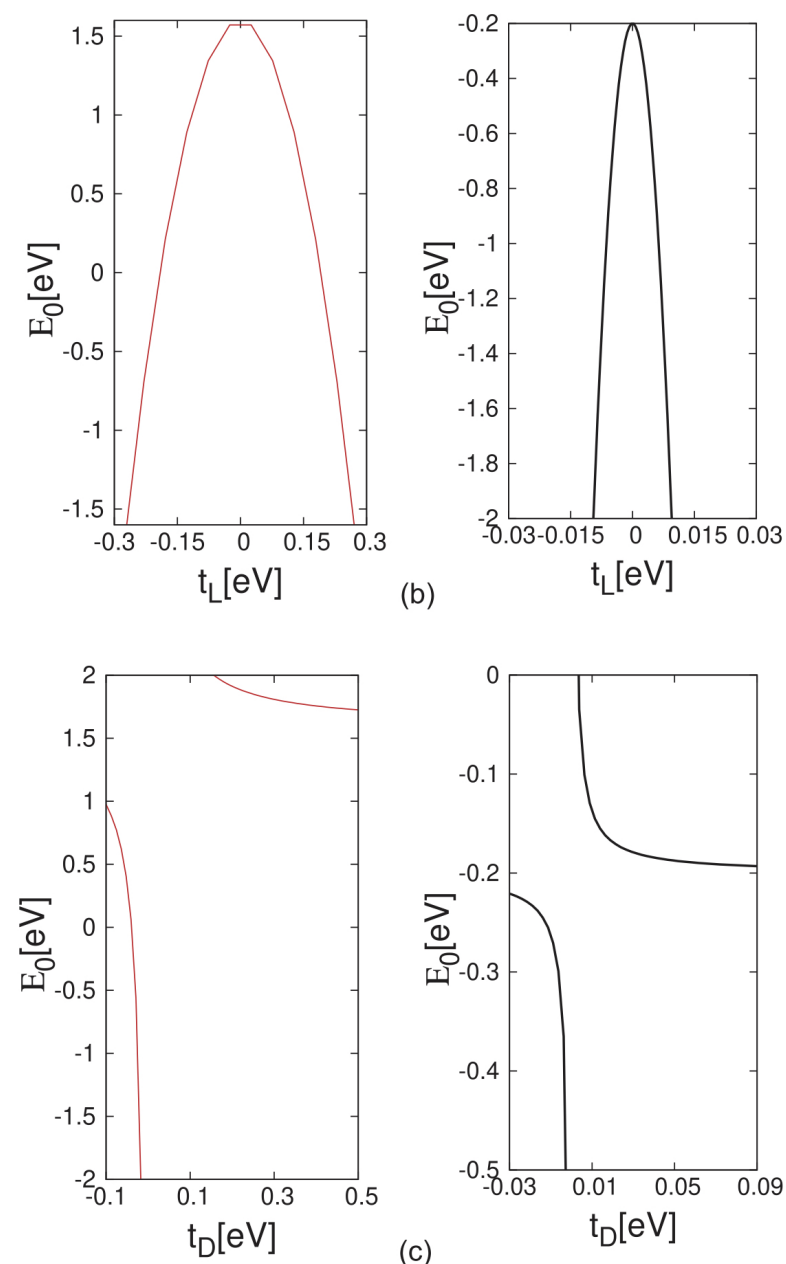

FIG. 8: $\mathrm{E}_{0}$ versus a) the energy difference $\Delta \varepsilon$ between the anchor and bridge states; b) the coupling value $\mathrm{t}_{L / R}$ and c) $\mathrm{t}_{D}$ for $\mathrm{Ru}$ (black curve) and $\mathrm{Fc}$ (red curve), respectively. In panel a) the $\mathrm{E}_{0}$ values resulting from $\Delta \varepsilon(\mathrm{Ru})$ and $\Delta \varepsilon(\mathrm{Fc})$ at -1.5 and $0.6 \mathrm{eV}$ are also marked as black and red dots, respectively. 


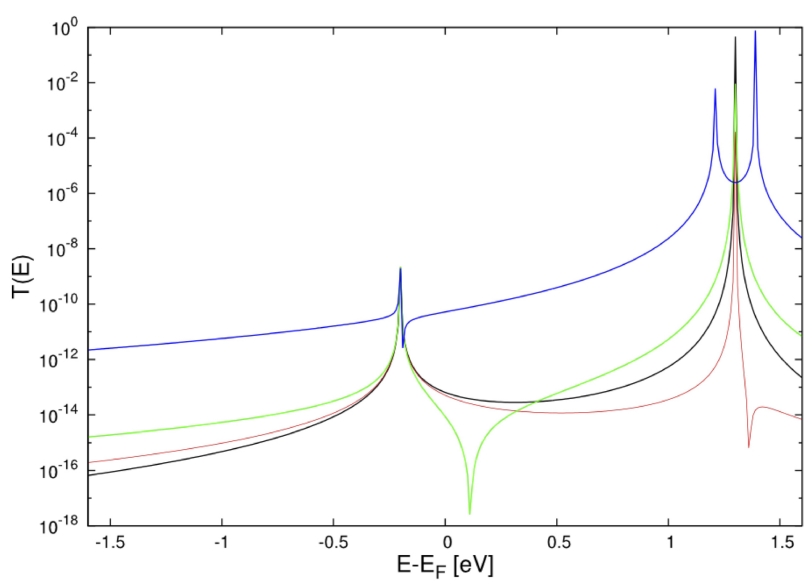

(a)

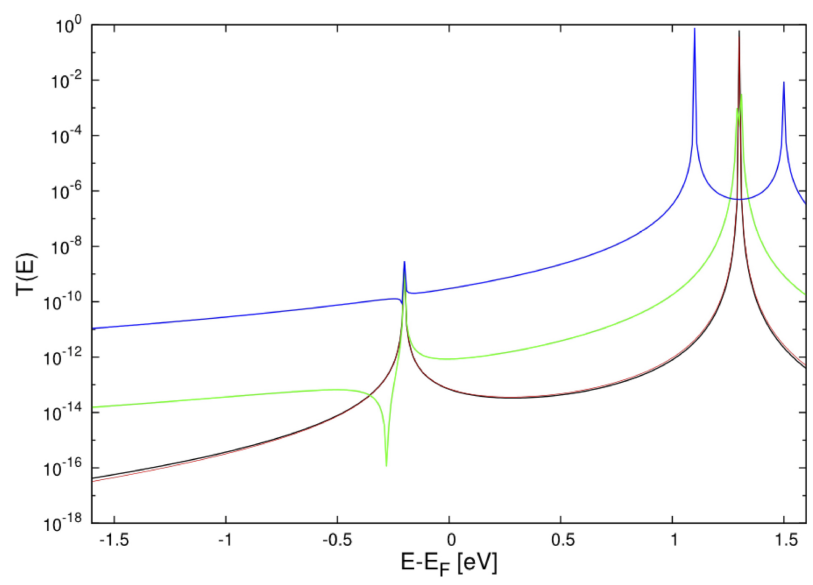

(b)

FIG. 9: Transmission functions calculated with NEGF-TB for the single-branched $\mathrm{Ru}$ system for a) $\mathrm{t}_{D}=0.00005 \mathrm{eV}$ (black line), $0.0004 \mathrm{eV}$ (red line), $0.002 \mathrm{eV}$ (green) and 0.09 $\mathrm{eV}$ (blue curve), and for $\mathrm{b}) \mathrm{t}_{D}=-0.00005 \mathrm{eV}$ (black line), $-0.0001 \mathrm{eV}$ (red line), $-0.008 \mathrm{eV}$ (green line) and $-0.2 \mathrm{eV}$

(blue line), respectively.

represented by the transport through the metal center via $\mathrm{t}_{L / R}$ and the transport from anchor to anchor mediated by $\mathrm{t}_{D}$ as also illustrated in Fig. 6. This seems to be a general rule regardless of the detailed quantitative values for $\Delta \varepsilon, \mathrm{t}_{L / R}$ and $\mathrm{t}_{D}$, which will facilitate the chemical design of molecules enabling DQI in their electron transport characteristics in the future.

\section{EFFECT OF CHARGING}

In order to ensure the charge neutrality in the unit cell of the system, which is necessary also for a junction with a charged molecule when applying periodic boundary conditions for electronic-structure calculations, the countercharge to the complex has to be an explicit part of the cell, where we use $\mathrm{Cl}^{-}$as a counterion (Fig. 10.

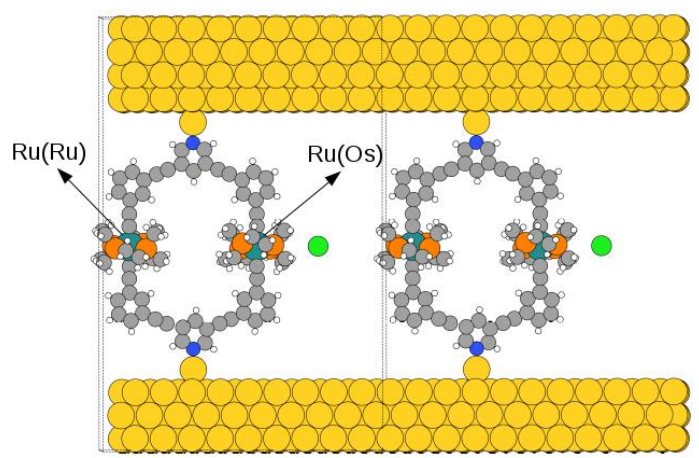

FIG. 10: Junction geometry for two neighbouring cells in the periodic setup for the scattering region of double branched $\mathrm{Ru} /$ Os containing a chlorine atom for achieving local charging with a distance of $5.2 \AA$ between $\mathrm{Cl}$ and the closer-lying metal atom.

We used the generalized $\Delta$ SCF method for calculating the charging effect in this section, where one additional electron on the chlorine $p$ shel ${ }^{30}$ is subtracted from the molecule. In this way the molecule is charged and we keep the neutrality of the unit cell in our calculations. This approach makes use of the flexibility of the generalized $\Delta$ SCF method to define the spatial expansion of an orbital which is forced to contain an electron as an arbitrary linear combination of Bloch states ${ }^{35136}$ and in our calculations is localized on a single $\mathrm{Cl}$ atom only as is predefined at the beginning of each iteration step. The self-consistency cycle then progresses as usual, but with the electron density of this particular orbital as a contribution to the external potential. In this way we can fix the electron occupation for the $\mathrm{Cl}$ manually, which solves the self-interaction problem implicitly and makes this method ideal for introducing localized charges into a junction 30 .

The redox centers with localized $d$ states on the metal atom in the investigated systems are stabilized by four donor ligands, which suggests a tetragonal ligand field 37 . According to Ligand field theory, for an octahedral field with Jahn Teller distortion, the orbitals $d_{x^{2}-y^{2}}$ and $d_{z^{2}}$ are in higher lying and the orbitals $d_{x z}, d_{y z}$ and $d_{x y}$ in

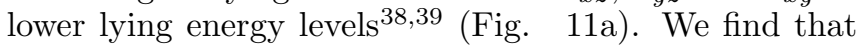
the peaks in the occupied region close to the Fermi level have contributions mostly from $d$ orbitals with $d_{x z}$ or $d_{y z}$ symmetries (Fig. 11b), where the $d_{x y}$ lies further below in energy (Fig. 111c). For the systems investigated here the $d$-orbitals $d_{x z}$ and $d_{y z}$ are fully occupied for all systems when in their neutral state, while for the charged state when $\mathrm{Ru}$ (II) is ixidized to $\mathrm{Ru}$ (III) (Fig. 11a), either $d_{x z}$ or $d_{y z}$ is singly occupied according to Hund's rule. This single occupation of a localized state leads 
(a)

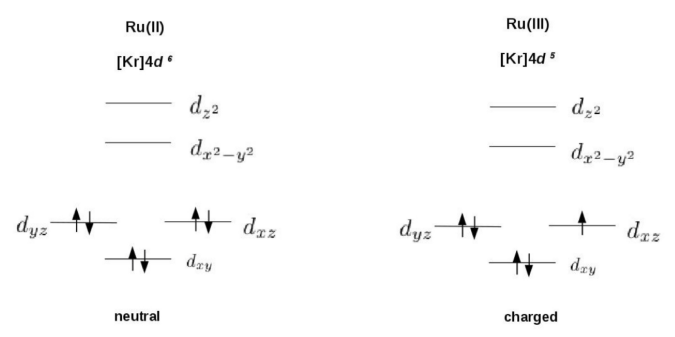

(b)

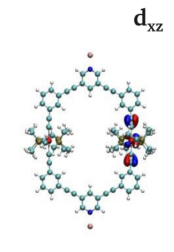

$\mathrm{d}_{\mathrm{xz}}$

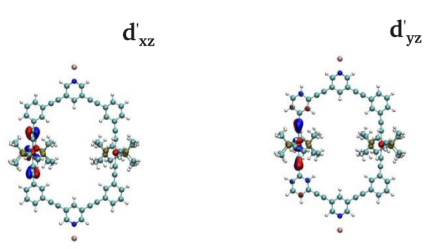

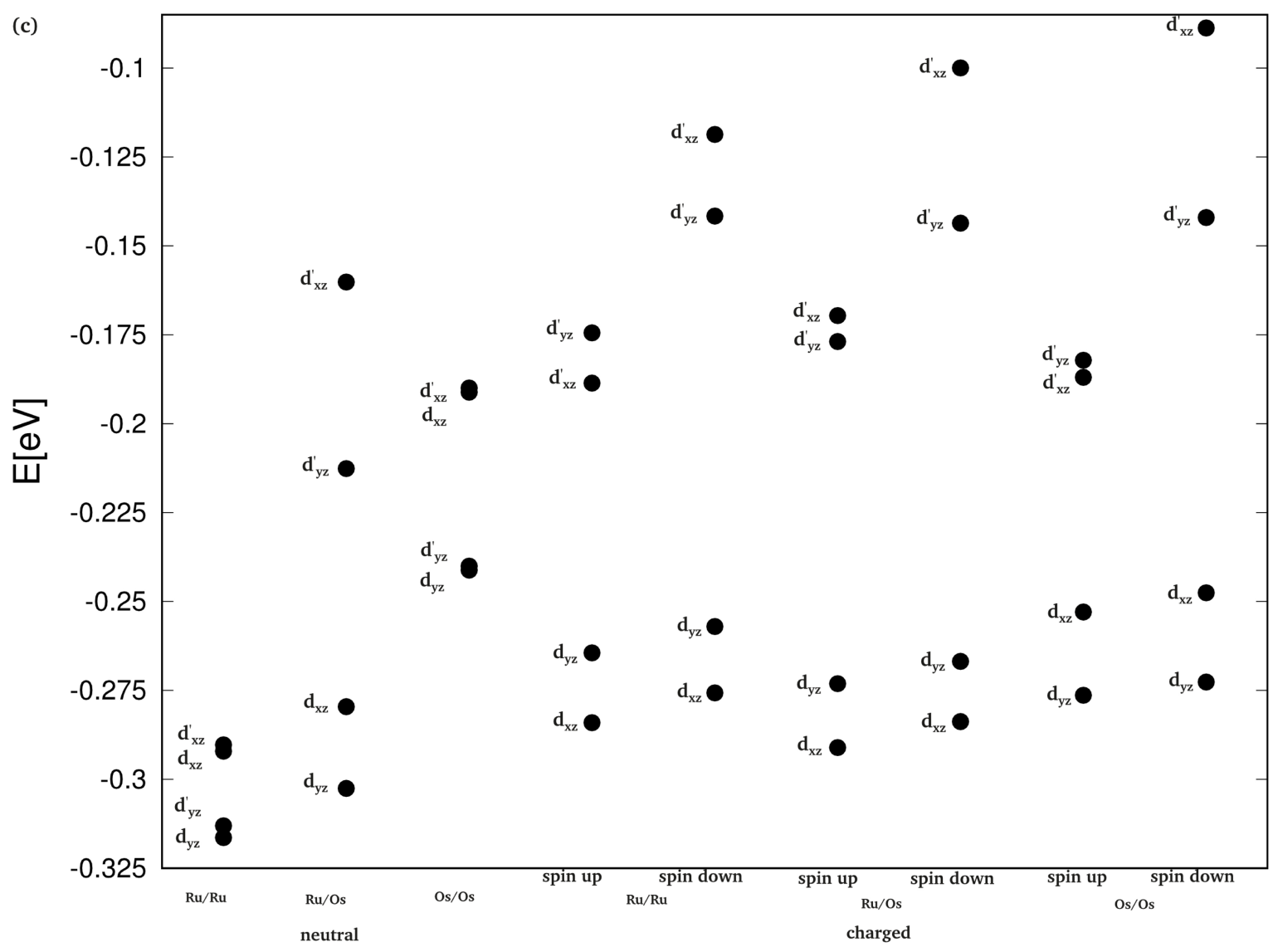

FIG. 11: a) Electron occupation of the Ru $d$ orbitals $\mathrm{n}$ a low-spin configuration for neutral and charged molecules according to ligand field theory, b) spatial localization of the four relevant occupied orbitals and c) their corresponding energies within the junction and with respect to the Fermi level for three systems, namely $\mathrm{Ru} / \mathrm{Ru}, \mathrm{Ru} / \mathrm{Os}$ and Os/Os in their neutral and charged states, respectively.

to the necessity of spin polarisation in our DFT calculations because spin-up and spin-down orbitals are then not equivalent in energy anymore.

From the respective MO eigenergies within the junction in Fig. 11k, which we obtained from a subdiagonalization of the transport Hamiltonian, it can be seen that for the asymmetric system $\mathrm{Ru} / \mathrm{Os}$ the amount of the shift induced by charging is smaller than for the two symmet- ric systems $\mathrm{Ru} / \mathrm{Ru}$ and $\mathrm{Os} / \mathrm{Os}$. For $\mathrm{Ru} / \mathrm{Os}$ also differs from the other two molecules in the energetic sequence of the orbitals, where there are no degeneracies for this case and no changes in sequence when moving from the neutral to the charged system. We also show the difference for the energies of the MOs on the branch closer to $\mathrm{Cl}$ and on the other branch with respective $d_{x z}$ and $d_{y z}$ symmetries in Table III] which confirms the same 

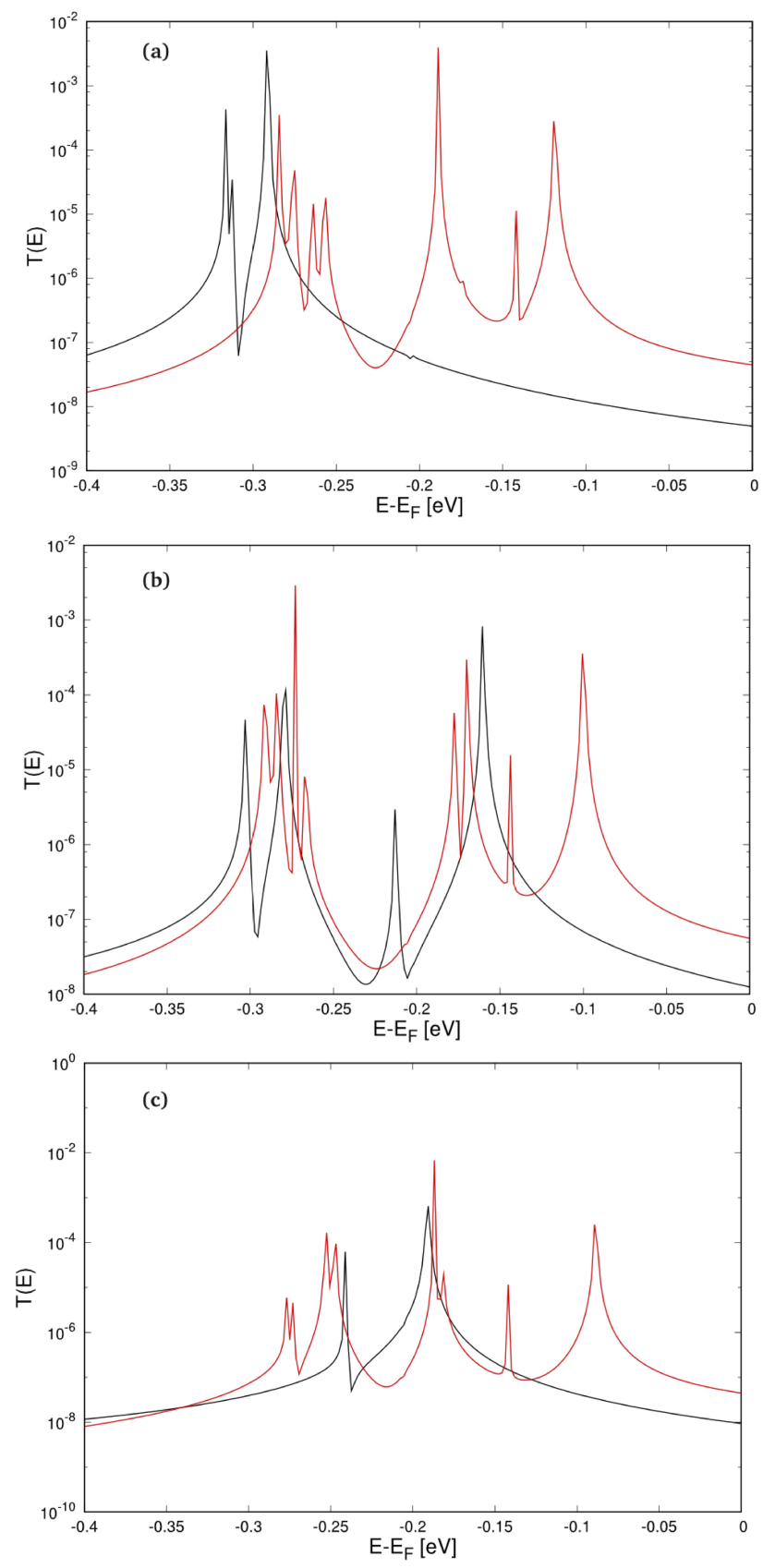

FIG. 12: Transmission functions for a) $\mathrm{Ru} / \mathrm{Ru}, \mathrm{b}) \mathrm{Ru} / \mathrm{Os}$ and c) Os/Os in their respective neutral (black solid line) and charged states (red solid line), where for the latter the average of spin-up and spin-down contributions has been used.

trends.

Fig. 12 shows the transmission functions for each branched molecule in this study $(\mathrm{Ru} / \mathrm{Ru}, \mathrm{Ru} / \mathrm{Os}, \mathrm{Os} / \mathrm{Os})$ in their respective neutral and charged states, where we conducted spin-polarized NEGF-DFT calculations for all charged systems. It can be seen that the peak splitting for the two symmetrically built molecules $\mathrm{Ru} / \mathrm{Ru}$ and
Os/Os after charging is pronounced, where MOs on the branch closer to the chlorine atom shifted more with respect to the Fermi level, and the MOs on the other branch almost have not been affected by the chlorine charging effect. For the asymmetrically built molecule $\mathrm{Ru} / \mathrm{Os}$ the peak splitting caused by charging is less distinct, because of the peak splitting already occuring in the neutral case due to the in-built asymmetry of the molecule, where charging does not seem to increase this splitting much further.

In Table IV we list the conductance of the neutral and charged states for each system as well as the partial charge on each molecule in order to investigate the sources of the asymmetry in MO energies as induced by charging and their effect on the coherent electron transport through the junction. While there is a marked difference between the partial charges on the two branches for the charged versions of all three molecules, the conductance of the charged systems changes only slightly when compared with their neutral counter parts, since the energy shifts of the peaks in the occupied region are relatively small and the conductance is dominated by those rather narrow peaks. Therefore, we conclude that these molecules are not suitable for redox switches, since although one of the two branches can be selectively oxidized this does not result in a significant DQI induced reduction of the conductance.

\section{SUMMARY}

In this study we investigated the potential use of branched molecules containing different metal centers in two branches as molecular transistors where the switching would be achieved by a redox process allowing to alternate between an ON and an OFF state, which would differ in their conductance by the occurrence of DQI effects in only one of these two redox states. We did, however, not find a DQI effect in the coherent electron transport through the branched molecules in our study, neither in their neutral nor in their charged states.

By comparing our results with previously studied ferrocene compounds, we further developed a scheme for the analysis of the structural conditions for the occurence or absence of DQI in branched metal complexes with redox active groups in each branch. We found that the ratio of the through-space coupling $\mathrm{t}_{D}$ and the couplings between anchor and bridge states $t_{L / R}$ play a decisive role in this context, which is signficant for chemical design purposes. These parameters, however, are barely altered by the oxidation of one of the two branches. As a consequence, the charging effect on the conductance of these cyclic molecules is not pronounced where only a rather moderate upward shift in energy of the narrow peaks in the transmission curves corresponding to occupied MOs on one branch is found.

Our findings and the analysis scheme we developed are likely to facilitate the design of DQI-based redox switches 
TABLE III: Energy differences in eV between the occupied d orbitals close to $\mathrm{E}_{F}$ with symmetries $\mathrm{d}_{x z}$ and $\mathrm{d}_{y z}$ localized on the two branches $\mathrm{M}_{1}$ and $\mathrm{M}_{2}$, where $\mathrm{M}_{2}$ is closer to the $\mathrm{Cl}$ atom and contains Os for the mixed case $\mathrm{Ru} /$ Os. For $\mathrm{M}_{1}$ we mark the respective $\mathrm{d}$ orbitals as $\mathrm{d}_{x z}$ or $\mathrm{d}_{y z}$ and for $\mathrm{M}_{2}$ as $\mathrm{d}_{x z}^{\prime}$ or $\mathrm{d}_{y z}^{\prime}$. We use the same notation as in Fig. 11 for all double branched molecules in their respective neutral and charged states. For all systems we define $\Delta \varepsilon\left(d_{x z}\right)=d_{x z}^{\prime}-d_{x z}$ and $\Delta \varepsilon\left(d_{y z}\right)=d_{y z}^{\prime}-d_{y z}$.

\begin{tabular}{cccc|ccc}
\hline \hline & \multicolumn{3}{c}{$\Delta \varepsilon\left(d_{x z}\right)$} & \multicolumn{3}{c}{$\Delta \varepsilon\left(d_{y z}\right)$} \\
\hline & $\mathrm{Ru} / \mathrm{Os}$ & $\mathrm{Os} / \mathrm{Os}$ & $\mathrm{Ru} / \mathrm{Ru}$ & $\mathrm{Ru} / \mathrm{Os}$ & $\mathrm{Os} / \mathrm{Os}$ & $\mathrm{Ru} / \mathrm{Ru}$ \\
\hline neutral & 0.120 & 0.001 & 0.002 & 0.090 & 0.001 & 0.003 \\
\hline charged/spin up & 0.121 & 0.064 & 0.098 & 0.096 & 0.094 & 0.090 \\
\hline charged/spin down & 0.184 & 0.159 & 0.157 & 0.123 & 0.131 & 0.115 \\
\hline
\end{tabular}

TABLE IV: Partial charges as obtained from a Bader analysis 40 for each of the two branches (metal complex plus all penyl and acetylene spacers but without the pyridyl anchors) of the double branched neutral and charged molecules, where $\mathrm{M}_{1}$ and $\mathrm{M}_{2}$ denote the branch containing the metal center further away from and closer to the chloride ion, respectively. All values for the charges are given in fractions of electrons. The conductance $\mathrm{G}$ for all molecules as defined by $\mathcal{T}\left(E_{F}\right)$ is given in units of $\mathrm{G}_{0}$.

\begin{tabular}{cccc}
\hline \hline & $\mathrm{M}_{1}$ & $\mathrm{M}_{2}$ & $\mathrm{G}$ \\
\hline \hline $\mathrm{Ru} / \mathrm{Ru}($ neutral) & -0.038 & -0.037 & $4.94 \times 10^{-9}$ \\
\hline $\mathrm{Ru} / \mathrm{Ru}(\mathrm{Cl})$ & -0.208 & -0.662 & $4.48 \times 10^{-8}$ \\
\hline \hline $\mathrm{Ru} / \mathrm{Os}($ neutral) & -0.0345 & -0.0378 & $1.26 \times 10^{-8}$ \\
\hline $\mathrm{Ru} / \mathrm{Os}(\mathrm{Cl})$ & -0.173 & -0.710 & $5.58 \times 10^{-8}$ \\
\hline \hline $\mathrm{Os} / \mathrm{Os}($ neutral) & -0.036 & -0.036 & $9.19 \times 10^{-9}$ \\
\hline Os/Os $(\mathrm{Cl})$ & -0.230 & -0.653 & $4.46 \times 10^{-8}$ \\
\hline \hline
\end{tabular}

and to interpret experimental observations on such complex molecules in the future.

\section{ACKNOWLEDGMENTS}

$\mathrm{XZ}$ and RS both have been supported by the Austrian Science Fund FWF (project number No. P27272). We are indebted to the Vienna Scientific Cluster VSC, whose computing facilities were used to perform all calculations presented in this paper (project No. 70671). We gratefully acknowledge helpful discussions with Georg Kastlunger.
* Email robert.stadler@tuwien.ac.at

1 M. Ratner, Nature Nanotech. 8, 378 (2013).

${ }^{2}$ E. Lörtscher, Nature Nanotech. 8, 381 (2013).

3 Y. Meir and N. S. Wingreen, Phys. Rev. Lett., 68, 2512 (1992).

4 M. Brandbyge, J. L. Mozos, P. Ordejon, J. Taylor and K. Stokbro, Phys. Rev. B 65, 165401 (2002).

5 Y. Xue, S. Datta and M. A. Ratner, Chem. Phys. 281, 151 (2002).

6 A. R. Rocha, V. M. Garcia-Suarez, S. W. Baily, C. J. Lambert, J. Ferrer and S. Sanvito, Nature Materials 4, 335 (2005).

7 K. S. Thygesen and K. W. Jacobsen, Chem. Phys. 319, 111 (2005).

8 C. Joachim, J.K. Gimzewski, R.R. Schlittler and C. Chavy, Phys. Rev. Lett. 74, 2102 (1995).

9 M.A. Reed, C. Zhou, C.J. Muller, T.P. Burgin and J.M. Tour, Science 278, 252 (1997).

10 J. Reichert, R. Ochs, D. Beckman, H.B. Weber, M. Mayor and H.v. Löhneysen, Phys. Rev. Lett. 88, 176804 (2002).
11 R.H.M. Smit, Y. Noat, C. Untiedt, N.D. Lang, M.C. van Hemert, and J.M. van Ruitenbeek, Nature (London) 419, 906 (2002).

12 M. Mayor, H. B. Weber, J. Reichert, M. Elbing, C. von Hänisch, D. Beckmann and M. Fischer, Angew. Chem. Int. Ed. 42, 5834 (2003).

13 C. J. Lambert, Chem. Soc. Rev. 44, 875 (2015).

14 R. Stadler, S. Ami, M. Forshaw and C. Joachim, Nanotechnology 15, S115 (2004).

15 R. Stadler, M. Forshaw and C. Joachim, Nanotechnology 14, 138 (2003).

16 R. Stadler and T. Markussen, J. Chem. Phys. 135, 154109 (2011).

17 C. M. Finch, V. M. Garcia-Suarez and C. J. Lambert, Phys. Rev. B 79, 033405 (2009).

18 C. M. Guedon, H. Valkenier, T. Markussen, K. S. Thygesen, J. C. Hummelen and S. J. van der Molen, Nat. Nanotechnol. 7, 305 (2012).

19 M. S. Inkpen, T. Albrecht and N. J. Long, Organometallics 32, 6053 (2013). 
20 G. Kastlunger and R. Stadler, Phys. Rev. B 91, 125410 (2015).

${ }^{21}$ F. Schwarz, G.Kastlunger, F. Lissel, H. Riel, K. Venkatesan, H. Berke, R. Stadler and E. Lörtscher, Nat Nanotechnol, 11, 170176 (2016).

${ }^{22}$ X. Zhao,G. Kastlunger, and R. Stadler, Phys. Rev. B 96, 085421 (2017).

23 J. P. Perdew, K. Burke and M. Ernzerhof, Phys. Rev. Lett. 77, 3865 (1996).

24 A.H. Larsen, M. Vanin, J.J. Mortensen, K.S. Thygesen and K.W. Jacobsen, Phys. Rev. B, 80, 195112 (2009).

25 J.J. Mortensen,L. B. Hansen and K.W. Jacobsen, Phys. Rev. B 71, 035109 (2005).

26 J. Enkovaara et al. Phys.: Conf. Ser. 22, 253202 (2010).

27 R. Stadler, K. S. Thygesen and K. W. Jacobsen, Phys. Rev. $B$ 72, 241401(R) (2005).

28 R. Stadler, Phys. Rev. B 80, 125401 (2009).

${ }^{29}$ X. Zhao, V. Geskin and R. Stadler, J. Chem. Phys. 146, 092308 (2017).

30 G. Kastlunger and R. Stadler, Phys. Rev. B, 88, 035418
(2013).

31 S. Larsson, J. Am. Chem. Soc. 103, 4034 (1981).

32 M. A. Ratner, J. Phys. Chem. 94, 4877 (1990).

${ }^{33}$ G. Kastlunger and R. Stadler, Phys. Rev. B 89, 115412 (2014).

34 P. Sautet and M.-L. Bocquet, Phys. Rev. B 53, 4910 (1996).

35 J. Gavnholt, T. Olsen, M. Engelund and J. Schiøtz, Phys. Rev. B, 78, 075441 (2008).

36 T. Olsen, J. Gavnholt and J. Schiøtz, Phys. Rev. B, 79, 035403 (2009).

37 J. Bendix, T. Birk, T. Weyhermuller, Dalton Trans., 2737 (2005).

38 C. E. Schäffer, C. Anthon and J. Bendix. Coordination Chemistry Reviews, Theory and Computing in Contemporary Coordination Chemistry 253, 575 (2009).

39 F. Schwarz, G. Kastlunger, F. Lissel, C. Egler-Lucas, S.N. Semenov, K. Venkatesan, H. Berke, R. Stadler and E. Lörtscher, Nat. Nanotech. 11,170 (2016).

40 R. S. Mulliken, J. Chem. Phys., 23, 1833 (1955). 